\title{
Metformin use in T2D patients with reduced kidney function: reassuring, more evidence needed
}

\author{
Anil Pareek $^{1}$ (I) $\cdot$ Shruti Dharmadhikari ${ }^{1} \cdot$ Ravi T. Mehta $^{1} \cdot$ Kumar Naidu $^{1}$ \\ Received: 4 November 2019 / Accepted: 27 May 2020 / Published online: 9 June 2020 \\ (C) Research Society for Study of Diabetes in India 2020
}

Dear Editor,

The recently published trial assessed major adverse cardiovascular events (MACE) among patients with diabetes and reduced kidney function who continued treatment with metformin or a sulfonylurea through well-designed retrospective cohort study [1]. Until recently, the use of metformin was restricted in patients with reduced kidney function due to concerns regarding risk of lactic acidosis. In 2016, the US FDA changed its guidance based on evidence regarding safety of metformin in patients with mild to moderate kidney disease; however, its effect on clinical outcomes in patients with reduced kidney function was unknown. In view of this background, the results of the present study are important and reassuring.

Hypoglycemia and HF are two important events that influence cardiovascular outcomes in diabetic patients, more so in those with kidney disease. Hypoglycemia in T2D patients is a frequent event and is associated with increased risk of cardiovascular events and all-cause mortality. Hypoglycemia is most commonly associated with the use of insulin secretagogues (primarily sulfonylureas) or insulin. Other risk factors include advanced age and cognitive impairment, renal dysfunction, duration of diabetes mellitus, and missed or irregular meals [2]. Both diabetes and kidney disease are major and independent risk factors for the development of HF [3]. In the unweighted cohort, there were substantially more patients with comorbid HF in sulfonylurea group which were adjusted with propensity matching. However, during the observation period, incidence of HF was not assessed, although hospitalization for acute MI, stroke, and TIA was assessed.

Anil Pareek

anil.pareek@ipca.com

1 Medical Affairs and Clinical Research Department, Ipca Laboratories Limited, Mumbai, India
Thus, to better understand the influence of hypoglycemia and $\mathrm{HF}$ on $\mathrm{CV}$ outcomes, there is need to additionally assess the following: (1) overall incidence of hypoglycemia along with its severity, (2) incidence of hypoglycemia in patients who experienced $\mathrm{CV}$ events, (3) incidence of $\mathrm{CV}$ events in the two groups after excluding patients with hypoglycemia, and (4) hospitalization due to HF.

Finally, as reported, it cannot be determined whether metformin is associated with a reduced risk or sulfonylureas are associated with an increased risk of MACE outcome. Welldesigned prospective studies will further reassure the use of metformin in T2D patients with reduced kidney function.

\section{Compliance with ethical standards}

Conflicts of interest The authors are employees of Ipca Laboratories Limited which markets anti-diabetic drugs.

\section{References}

1. Roumie CL, Chipman J, Min JY, et al. Association of treatment with metformin vs sulfonylurea with major adverse cardiovascular events among patients with diabetes and reduced kidney function. JAMA. 2019;19:1-11.

2. Connelly KA, Yan AT, Leiter LA, Bhatt DL, Verma S. Cardiovascular implications of hypoglycemia in diabetes mellitus. Circulation. 2015;132(24):2345-50.

3. Gilbert RE, Connelly K, Kelly DJ, Pollock CA, Krum H. Heart failure and nephropathy: catastrophic and interrelated complications of diabetes. Clin J Am Soc Nephrol. 2006;1(2):193-208.

Publisher's note Springer Nature remains neutral with regard to jurisdictional claims in published maps and institutional affiliations. 